\title{
Modeling Incompressible Fluids by Means of the SPH Method: Surface Tension and Viscosity
}

\author{
Paweł Wróblewski ${ }^{1}$, Krzysztof Boryczko ${ }^{1}$, and Mariusz Kopeć ${ }^{2}$ \\ ${ }^{1}$ Department of Computer Science \\ AGH University of Science and Technology, Kraków \\ \{pawel.wroblewski, boryczko\} @agh.edu.pl \\ ${ }^{2}$ Faculty of Physics and Applied Computer Science \\ AGH University of Science and Technology, Kraków \\ makopec@agh.edu.pl
}

\begin{abstract}
The adaptations of the SPH method for simulating incompressible fluids which focuse on two features: the surface tension and artificial viscosity, are presented in this article. The background and principles of the SPH method are explained and its application to incompressible fluids simulations is discussed. The methodology and implementation of artificial viscosity in the SPH method are presented. The modification for surface tension simulation, which relies on incorporating additional forces into the model, as well as the methodology are suggested. Also, the new equations for artificial viscosity are presented, which are able to simulate a flow of non-newtonian fluids. The results obtained with the method are presented and discussed.
\end{abstract}

\section{Introduction}

A number of existing computer methods can be used for simulating phenomena from the real world. Many of these phenomena, very important in the contemporary science and engineering, are related to fluid mechanics. These phenomena refer to different spatio-temporal scales, starting from micro scale, through meso scale to macro scale. Very interesting processes, e.g. turbulences, wall-fluid interactions, free surface behavior, take place in the domain between these scales. However, neither correct nor efficient methods have been available for this area yet. The SPH method is a very popular particle method for simulating processes in the macro scale [12; it also seems to be possible to simulate phenomena from the domain between macro and meso scales. However, despite many advantages, the proposed method has also several drawbacks. From the authors' point of view, one of the most awkward one is the lack of possibility to simulate surface tension. There are a few papers presenting modifications of the SPH method which remove this disadvantage 16] 13. However, the analysis of these modifications reveals that they are either deprived of a strong physical background or are too complicated for computer implementation. Another drawback of the $\mathrm{SPH}$ method is the problem with modeling a flow of non-newtonian fluids. This topic is almost not present in papers concerning SPH simulations [15] and there 
is no straight scheme for obtaining the eligible model of non-newtonian viscosity, what opens this research area for new investigations.

The background and principles of SPH method are shown along with its variants, depending on the target application. There are also presented adaptations of this method to incompressible fluids simulations. The proposed modification of the SPH method enables modeling surface tension in several physical phenomena. The changes rely on additional forces acting between SPH particles as well as between SPH particles and walls of a vessel. The methodology of adding new forces is discussed. The implemented modified algorithm has been employed for simulating two phenomena: a fluid drop behavior in vacuum without gravity and a capillary rise between two vertical, parallel plates inserted into a fluid.

The second application of the SPH method presented in this paper is the simulation of a flow of non-newtonian fluid. In order to achieve it we propose new equations for artificial viscosity, which actually are a modification of Monaghan's artificial viscosity model. This modification consists of the change of the character of viscosity's dependence on interparticle velocity. In the proposed model this dependence is non-linear. The new model of viscosity was validated in the simulation of the flow of the viscous fluid in a long, cylindrical vessel. The non-newtonian character of the fluid manifested itself in the modified velocity profile for the modeled flow [4].

\section{Smoothed Particle Hydrodynamics}

The SPH method was created in order to simulate astrophysical phenomena [10] 5. The main idea of the method is a field approximation in the set of points from space. The hydrodynamical forces, corresponding to the Navier-Stoke's equations are calculated in these points (the SPH particles) and with such a background the equations of motion are solved. The approximation procedure uses the kernel function, which vanishes at infinity and its integral is equal to unity. From the theoretical point of view one could choose the gaussian bellshaped function, however in practice it is common to use the spline function with compact support. The authors present results obtained by means of the kernel function proposed in 11. The approximation procedure applies not only to the hydrodynamical forces, but also other quantities referred to by the modeled phenomenon. The approximation equation for a density is presented below [1]:

$$
\rho_{i}=\sum_{j} m_{j} W_{i j},
$$

where $m_{j}$ - the mass of particle $j, W_{i j}=W\left(r_{i j}, h_{i j}\right)$ - the kernel function evaluated for particles $i$ and $j, r_{i j}$ - the distance between particles $i$ and $j$ and $h_{i j}-$ a smoothing length. The sum in the above equation runs over all particles from the system. In practice, however, if the support of kernel function is compact, it is enough to count only those SPH particles, for which the kernel function is non-zero. Then, the character of interparticle interactions is shortrange; there exists cut-off radius $r_{c u t}$ such, that for every pair of particles whose 
distance is larger than $r_{c u t}$, the force acting between them is equal to zero. For simulation with short-range interactions it is possible to use the structure of cubic cells, which rapidly accelerate calculations [2].

Every SPH particle undergoes the acceleration given by the formula:

$$
\frac{\mathrm{d} \mathbf{v}_{i}}{\mathrm{~d} t}=-\sum_{j} m_{j}\left(\frac{P_{i}}{\rho_{i}^{2}}+\frac{P_{j}}{\rho_{j}^{2}}+\Pi_{i j}\right) \nabla_{i} W_{i j},
$$

where $P_{i}$ - the pressure at point $i, \rho_{i}$ - the density at point $i$ and $\Pi_{i j}$ - the viscosity part of the force. One can find the full derivation of equation (2) in 8]. Besides the force acting between fluid particles, it is also necessary to incorporate into the model forces acting between fluid particles and the walls. In this paper we use a wall consisting of particles, and the corresponding force is very similar to the one given in 12 . It is given by formula:

$$
\frac{\mathrm{d} \mathbf{v}_{i}}{\mathrm{~d} t}=\sum_{j}\left(\frac{c_{0}}{10}\right)^{2} \frac{\Gamma\left(r_{i j} / r_{c u t}\right)}{r_{i j}} \frac{m_{j}}{m_{i}+m_{j}},
$$

where

$$
\Gamma(q)= \begin{cases}\frac{2}{3}, & \text { if } q<\frac{1}{3} \\ 2\left(2 q-3 q^{2}\right), & \text { if } \frac{1}{3}<q<\frac{1}{2} \\ 2(1-q)^{2}, & \text { if } \frac{1}{2}<q<1 \\ 0, & \text { otherwise }\end{cases}
$$

\section{Incompressible $\mathrm{SPH}$}

The standard SPH formula for density (11) is useless when modeling the fluids with free surface. If this equation is applied, the density in the vicinity of the surface changes continuously from the value assumed for all particles to the value of zero on the distance of $2 h$, which is obviously a discord with the experiments. In the case of such fluids another formula derived from the SPH approximation is used:

$$
\frac{\mathrm{d} \rho_{i}}{\mathrm{~d} t}=\sum_{j} m_{j}\left(\mathbf{v}_{i}-\mathbf{v}_{j}\right) \cdot \nabla_{i} W_{i j},
$$

which evaluates only the rates of change of the density. Application of this equation requires the initialization of the density values at the beginning of the simulation.

The incompressible character of the fluid is modeled by an appropriate equation of state, which is used for evaluating the pressure values in equation (2). The authors use the equation of state given by [12]

$$
P=\frac{\rho_{0} c_{0}^{2}}{7}\left(\left(\frac{\rho}{\rho_{0}}\right)^{7}-1\right) \text {. }
$$


When modeling incompressible fluids it is very problematic to choose the proper timestep. If real values of speed of sound $c$ are applied, the timestep is too small for any practical application. Therefore it is convenient to use a value of $c$ several orders of magnitude smaller than the real one. This approach accelerates the calculation significantly, and does not influence results [3].

\section{Modeling Viscosity with SPH}

In many simulations of fluid flow it is necessary to comply the transition of kinetic energy of the fluid into its thermal energy. In the case of the SPH method presented here, where the thermal energy of the fluid is not concerned, it is necessary to incorporate the dissipation of the fluid energy by means of viscosity. Also, the every day experience of viscosity in almost all real fluids demands incorporating the viscosity term $\Pi_{i j}$ into the equation (2).

The most often used [8] model of artificial viscosity in SPH simulations is the one proposed by Monaghan [1]:

$$
\Pi_{i j}=\left\{\begin{array}{ll}
\frac{-\alpha \bar{c}_{i j} \mu_{i j}+\beta \mu_{i j}^{2}}{\bar{\rho}_{i j}} & , \text { if } \boldsymbol{v}_{i j} \cdot \boldsymbol{r}_{i j}<0 \\
0 & , \text { if } \boldsymbol{v}_{i j} \cdot \boldsymbol{r}_{i j} \geq 0
\end{array},\right.
$$

where

$$
\mu_{i j}=\frac{h \boldsymbol{v}_{i j} \cdot \boldsymbol{r}_{i j}}{r_{i j}^{2}+\eta^{2}} .
$$

Monaghan proposes to set $\eta^{2}=0.01 h^{2}$. In this model viscosity vanishes, when $\boldsymbol{v}_{i j} \cdot \boldsymbol{r}_{i j} \geq 0$, which has an equivalent on the SPH interpolation level: $\nabla \cdot \boldsymbol{v} \geq 0$ 11. According to this, viscosity is present only when two particles approach to each other. In the opposite case, the viscosity force is equal to 0 .

There are also other models of artificial viscosity used in SPH simulations. One can mention ones proposed by Hernquist and Katz [6] or by Balsara [1] A more detailed discussion of the appropriate choice of the artificial viscosity model is presented in [9. In simulations presented in this article the authors use the model proposed by Monaghan. Its advantages are: simplicity and relatively low computational costs.

\section{The Proposed Modifications of SPH Method for Simulating: Surface Tension and Non-newtonian Character of a Fluid}

\subsection{Additional Forces for Surface Tension Model}

Modeling the phenomena in which the surface tension effects arise one needs to incorporate two additional parts into the model. The first one represents interactions acting between fluid particles responsible for modeling the surface tension of the fluid. The second part is the modification of the fluid-wall interactions, which is responsible for proper modeling the wetting character of the fluid. 


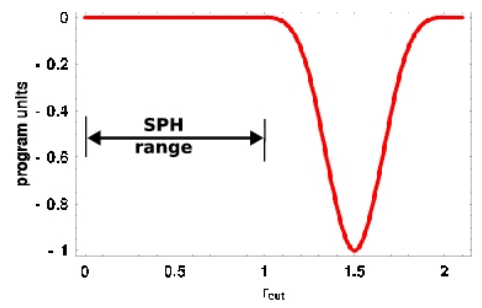

Fig. 1. The function of new additional force

Fluid-fluid interactions. The surface tension is an effect of mutual attraction of fluid molecules. It is impossible to simulate the exact inter-molecule interaction in the SPH method, because the scale of the method is much larger than the scale where intermolecule forces are present. However, the main idea of the model is still the same and it is realized by incorporating additional attractive forces. When trying to find the form of this force the authors found that it was very difficult to do so, when the range of the new, additional attractive force was the same as the range of the SPH interactions. In this case the additional force modified the nature of the SPH force, and together they led to numerical artifacts. For example, the SPH particles tended to bind in pairs. This is why, following the advice given in [14], the authors move the range of acting of the additional force beyond the SPH range. Actually, it is twice as far as SPH range. In this case the artifacts are not observed anymore, and the results are in good agreement with expectations.

The form of the new, proposed force is given by the equation:

$$
F_{i j}=-A \cdot W\left(\frac{3}{2} r_{c u t}-r_{i j}, \frac{1}{4} r_{c u t}\right),
$$

where $A$ is a positive constant and $W$ is the kernel function. The plot of this new function is depicted in the Fig. 1. The form of the proposed additional force is one of many possible. During tests with many different forms of this force the authors concluded, that, in general, the form of the force does not influence the results, if only values of the force are negative in the range $\left[r_{\text {cut }}, 2 r_{\text {cut }}\right]$. Therefore, the authors proposed the force given by (9), which is convenient to be implemented.

Fluid-wall interactions. Similarily, in order to model phenomena which concern hydrophilic or hydrophobic fluids it is also necessary to incorporate additional attractive forces acting between fluid particles and wall particles. In the original SPH model the force acting between walls and fluid was purely repulsive. The authors incorporated additional, attractive forces into the simulation. Their form is given by the formula (9), i.e. it is exactly the same, as in the case of fluid-fluid interactions, but with a different value of constant $A$. The reasons, why this particular form of the force was used are the same, as in the case mentioned above. 


\subsection{The Modification of Artificial Viscosity Model}

Additionally, in order to propose the model capable of simulating flows of nonnewtonian fluids, we propose a modification of Monaghans' artificial viscosity. The modification relies on a change of the equation (8) to:

$$
\mu_{i j}=\frac{h \boldsymbol{v}_{i j} \cdot \boldsymbol{r}_{i j}}{r_{i j}^{2}+\eta^{2}} \cdot \frac{\exp v_{i j}-1}{v_{i j}} .
$$

According to this change, an artificial viscosity acting between two particles depends nonlinearly on their mutual velocity, and this way it is possible to obtain the non-netwonian character of a fluid flow. This manifests itself in the change of the velocity profile of the flow, which now corresponds to the viscosity coefficient nonlinearly dependent on the shear rate [4].

By using modification given by (10) the authors introduced non-linear dependence of $\mu_{i j}$ on $v_{i j}$. The equation (10) is only an example of such modifications and is supposed to show the possibilities of further research in this area. The authors tested also several other equations and received similar results.

\section{Results}

The modifications presented above have been tested in simulations of three different fluid phenomena.

\subsection{Fluid Drop Oscillations without Gravity}

The first phenomenon used for validating the form of additional attractive forces was the behavior of fluid drop in vacuum. We took well equilibrated circular drop and transformed it into an ellipsoid with the transformation [14]:

$$
\left(\begin{array}{l}
x^{\prime} \\
y^{\prime}
\end{array}\right)=\sqrt{\frac{2}{\sin \phi}} r\left(\begin{array}{c}
\sin (\phi / 2) \sin u \\
\cos (\phi / 2) \cos u \operatorname{sgn} y
\end{array}\right),
$$

where $r=\sqrt{x^{2}+y^{2}}, \phi=0.63 \pi$ and $u=\arctan \frac{x}{y}$. The ' $z$ ' coordinate for every particle remained unchanged.

Then, we examined the relaxation time of the drop, which should depend on the surface tension value of the fluid. If there is no artificial viscosity, then the drop deformed with the above formula oscillates about the equilibrium state, which is ideal sphere. However, when the artificial viscosity is present, it is more convenient to examine the relaxation time. The sample relaxation scheme is depicted in the Fig. 2. The authors ran four different simulations, for four different values of the parameter corresponding to the surface tension value for the fluid. The results obtained are presented in the Fig. 3.

The relaxation time should depend on the surface tension coefficient $\gamma$ as $\sim \gamma^{-\frac{1}{2}}[14$. The plot presented in the Fig. 3] shows, that the results from the simulation are in good agreement with this dependence at least qualitatively. 


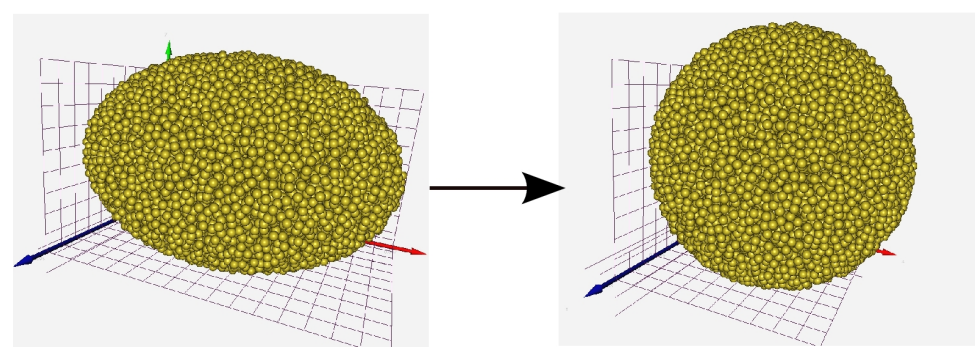

Fig. 2. A relaxation-oscillation of fluid drop in vacuum

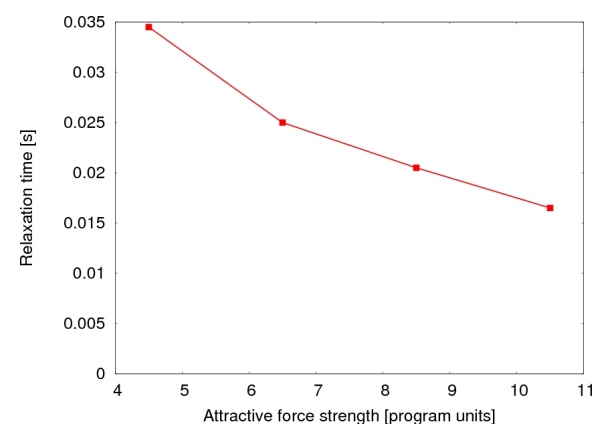

Fig. 3. A relaxation time versus surface tension

\subsection{Capillary Rise of the Liquid}

The second phenomenon used for validating the model of surface tension with the SPH method is capillary rise of the modeled liquid. The effect in this phenomenon depends on the difference between attractive forces of fluid-fluid interactions, and fluid-walls interactions. If fluid-fluid attractive forces are stronger than fluid-walls interactions, then one should expect to see convex meniscus (negative capillary rise). In the opposite case, a concave meniscus should be visible (positive capillary rise).

This is what the authors received from the simulations as a result. For fluidfluid attractive forces stronger than fluid-walls interactions, the results are as presented in the Fig. 4 a. On the other hand, when the fluid-wall interactions are stronger than the fluid-fluid ones, the obtained results are as in the Fig. 4.b.

Both simulated phenomena prove that the proposed modification of SPH method properly model the surface tension effects.

\subsection{The Fluid Flow in an Elongated Vessel}

The next phenomenon modeled by means of SPH method is the fluid flow in an elongated vessel. In the beginning all fluid particles filling the vessels rested. Then we applied the initial velocity to all of them and continued the simulation till the flow stopped. The flow was delayed by the forces acting between the 
a)

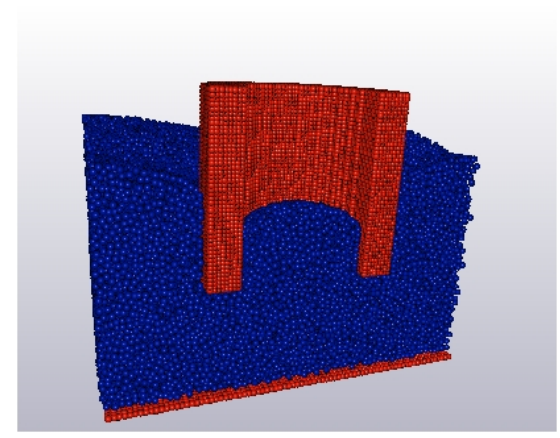

b)

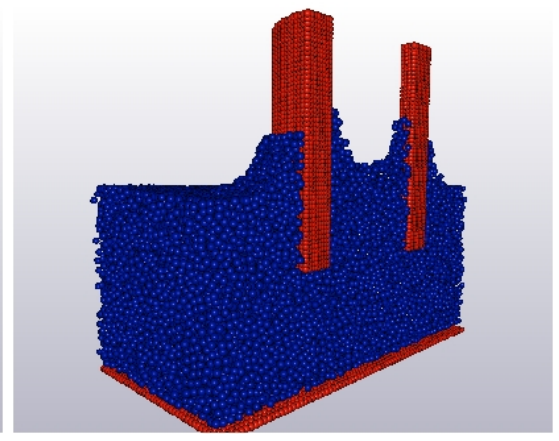

Fig. 4. Two menisci obtained from the simulations: a) convex meniscus, b) concave meniscus

a)

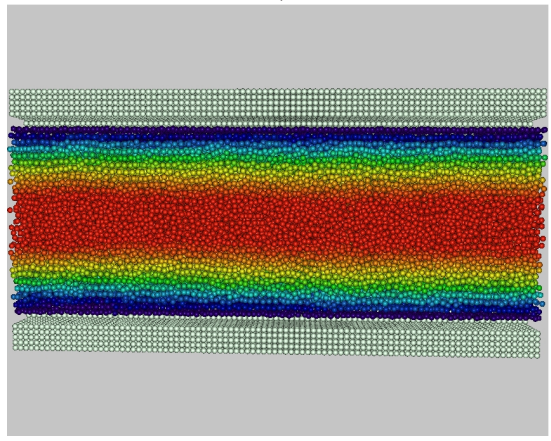

b)

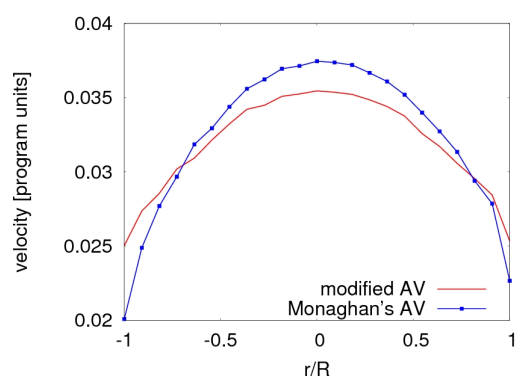

Fig. 5. a) Velocity distribution. b) Velocity profiles for two different viscosity models.

wall and fluid particles. The interactions of wall-fluid particles were modeled by means of the DPD method [7], where brownian part of the force was omitted. It was the dissipative part of the DPD model, which delayed flow of a fluid. The sample velocity distribution, where velocities were marked with color, is presented in the Fig. 5]a. The authors ran several such simulations, each for different artificial viscosity model. When compared the Monaghan's model with the modified artificial viscosity, it seems that our modification can be treated as a starting point for further research on the area of non-newtonian fluid flows. This can be derived from the analysis of velocity profiles from executed simulations. The velocity profiles of two models: Monaghan's model and the one given by equation (10), are presented in the Fig. 5]b. The obtained profile is more flattened than the Monaghan's one, which meets the expectations 4].

\subsection{The Parallel Implementation of the Model}

The SPH method, along with presented modifications, was implemented in parallel by means of the OpenMP environment. The simulation of a fluid flow was 


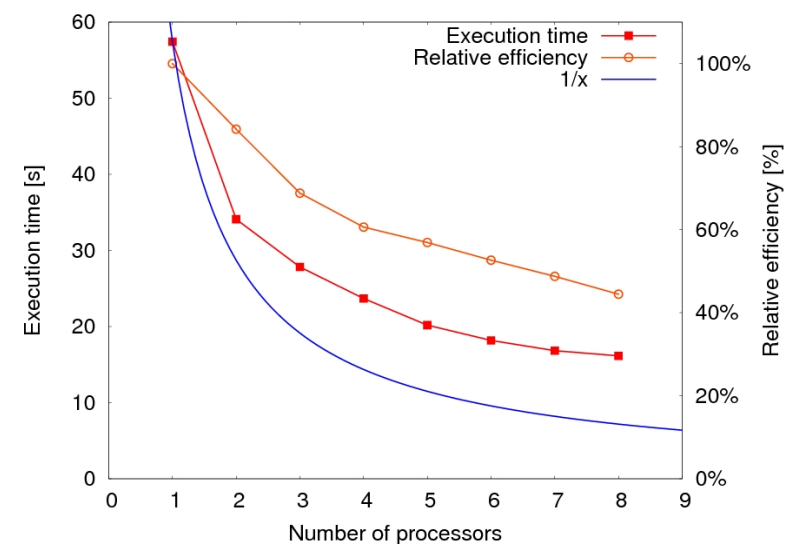

Fig. 6. The relative efficiency of OpenMP implementation

ran for several values of the number of processors. The time of execution of a single simulation step was measured, and the relative efficiency was evaluated. The results are depicted in the Fig. 6. The similar values of relative efficiency were obtained for simulation of SPH method with modifications for surface tension. In this case, however, the execution time of a single simulation step was greater, since the interparticle interaction range was doubled.

\section{Conclusions and Future Work}

The results presented in the paper show that the proposed form of the additional forces in the SPH method properly model the surface tension of the simulated fluid. These forces were validated in two simulated phenomena, and satisfactory results were obtained.

Also, the simulation of the fluid flow with the modified artificial viscosity indicates that proposed improvements allow for reasonable modeling of nonnewtonian fluids. Such simulations will find different applications in science and engineering, for example in modeling properties of the blood flows 44. However, this method still needs additional work and more detailed derivation of its equations.

The presented results qualitatively prove the correctness of the modified method. However, it is still a big challenge to validate the new forces quantitatively. Yet, no analytical relation between physical quantities such as viscosity, surface tension and simulation parameters is known.

Acknowledgments. This research is financed by the Polish Ministry of Science and Higher Education, Project No. 3 T11F 01030. 


\section{References}

1. Balsara, D.: Von Neumann stability analysis of smoothed particle hydrodynamicsSuggestions for optimal algorithms. J. Comput. Phys. 121, 357 (1995)

2. Boryczko, K., Dzwinel, W., Yuen, D.: Parallel implementation of the fluid particle model for simulating complex fluids in the mesoscale. Concurrency and computation: practice and experience 14, 137-161 (2002)

3. Colagrossi, A., Landrini, M.: Numerical simulation of interfacial flows by smoothed particle hydrodynamics. J. Comp. Phys. 191, 448-475 (2003)

4. Gijsen, F., Vosse, F., Janssen, J.: The influence of the non-newtonian properties of blood on the flow in large arteries: steady flow in a carotid bifurcation model. Journal of Biomechanics 32, 601-608 (1999)

5. Gingold, R.A., Monaghan, J.J.: Smoothed particle hydrodynamics - Theory and application to non-spherical stars. Mon. Not. R. Astr. Soc. 181 (1977)

6. Hernquist, L., Katz, N.: TREESPH: A unification of SPH with the hierarchical tree method. The Astrophysical Journal Supplement Series 70, 419-446 (1989)

7. Hoogerbrugge, P.J., Koelman, J.: Simulating microscopic hydrodynamic phenomena with dissipative particle dynamics. Europhys. Lett. 19, 155-160 (1992)

8. Liu, G.R., Liu, M.B.: Smoothed particle hydrodynamics: a meshfree particle method. World Scientific, Singapore (2003)

9. Lombardi, J., Alison, S., Rasio, F., Shapiro, S.: Tests of Spurious Transport in Smoothed Particle Hydrodynamics. Journal of Computational Physics 152, 687735 (1999)

10. Lucy, L.B.: A numerical approach to the testing of the fission hypothesis. Astron. J. 82, 1013-1024 (1977)

11. Monaghan, J.J.: Smoothed Particle Hydrodynamics. Annu. Rev. Astron. Astrophys. 30, 543-574 (1992)

12. Monaghan, J.J.: Smoothed Particle hydrodynamics. Rep. Prog. Phys. 68, 17031759 (2005)

13. Morris, J.P.: Simulating surface tension with smoothed particle hydrodynamics. Int. J. Numer. Meth. Fluids 33, 333-353 (2000)

14. Nugent, S., Posch, H.A.: Liquid drops and surface tension with smoothed particle applied mechanics. Phys. Rev. E 62, 4968-4975 (2000)

15. Shao, S., Lo, E.Y.M.: Incompressible SPH method for simulating Newtonian and non-Newtonian flows with a free surface. Advances in Water Resources 26, 787-800 (2003)

16. Tartakovsky, A., Meakin, P.: Modeling of surface tension and contact angles with smoothed particle hydrodynamics. Phys. Rev. E 72, 02630 (2005)

17. Wróblewski, P., Boryczko, K., Kopeæ, M.: SPH - a comparison of neighbor search methods based on constant number of neighbors and constant cut-off radius. TASK Quart. 11, 275-285 (2007) 